rung begründet und nicht - woran man denken könnte - aus der Sozialgebundenheit des Eigentums hergeleitet werden.

Grundpfandrechte sind nach den Ausführungen des Verfassers offenbar nur in der Form der Verknüpfung von persönlicher Schuld und dinglicher Sicherheit bekannt; anscheinend sind nichtakzessorische Rechte wie die Grundschuld nicht existent.

Das Buch schließt mit einem Kapitel über Neuerungen im Immobiliarrecht, es erläutert die großen Landreformen in Ägypten, Syrien und im Iraq. Ein Anhang geht summarisch auf die Rechtslage in Saudi-Arabien und in den Golfstaaten ein und weist die allerdings sehr begrenzten Möglichkeiten für Ausländer auf, Grundeigentum zu erwerben.

Dagmar Hohberger

\title{
Hasan Moinuddin
}

Die Organisation der Islamischen Konferenz (OIC) als Forum politischer und wirtschaftlicher Kooperation

Studienverlag Brookmeyer, Sozialwissenschaftliche Studien, Bd. 24, Bochum, 1984, 214 S., DM 29,80

1969 wurde in Rabat auf der ersten islamischen Gipfelkonferenz die Organisation der Islamischen Konferenz (OIC) gegründet, deren Charta 1973 in Kraft trat.

In seiner 1984 vorgelegten Dissertation arbeitet Hasan Moinuddin die Charakteristika dieser Organisation heraus, wobei er insbesondere untersucht, inwieweit die universalen Grundsätze des Völkerrechts beachtet werden. Hierbei kommt er zu dem Ergebnis, daß die Prinzipien Souveränität, Gleichheit der Staaten, Selbstbestimmung und Interventionsverbot, friedliche Streitbeilegung sowie Gewaltverbot anerkannt werden, obwohl sie zum Teil zur klassischen Djihaddoktrin in Widerspruch stehen. Während seine Darstellung der Djihaddoktrin und ihre Bedeutung bzw. Abwandlung in der Gegenwart gelungen ist, finden sich bei der Behandlung der völkerrechtlichen Prinzipien doch manche Ungenauigkeiten (so etwa bei der Erörterung des Gleichheitssatzes und seinen Auswirkungen auf die Entscheidungsfindung S. 49 ff.).

In weiten Passagen des Werks erörtert der Verfasser die einzelnen Bestimmungen der Charta. Dies geschieht jedoch sehr oberflächlich und stellt oftmals nur eine Wiedergabe des Textes dar. Eingegangen wird auf das Problem, welcher Staat als wislamischer Staat" zu verstehen ist. Der Entwurf der Charta sah eine Differenzierung zwischen Vollmitgliedschaft und Teilnehmerstatus vor. Die Charta in der ratifizierten Form trifft diese Unterscheidung nicht mehr, vielmehr besteht die Möglichkeit, beim Beitritt einen Vorbehalt zu erklären. Zur Zeit gehören 44 Staaten der OIC an.

Eine Bewertung der OIC ist angesichts der geringen praktischen Aktivität schwierig. So fanden nach Inkrafttreten der Charta lediglich 1974 in Lahore, 1981 in Mekka und 1984 in Casablanca Gipfelkonferenzen statt. Es wurde nunmehr vereinbart, jeweils Gipfelkonferenzen im 3-Jahres-Turnus abzuhalten. 
Aus der bisherigen Praxis geht der Verfasser näher auf die Suspendierung der Mitgliedschaft von Afghanistan (Reaktion auf die politische Präsenz der UdSSR) sowie Ägyptens (nach dem Friedensvertrag von Camp David) ein. Die Charta selbst sieht einen Ausschluß nicht vor. Unter Berufung auf allgemeines Völkerrecht sowie Art. 60 Wiener Vertragsrechtskonvention hält Moinuddin dennoch die Suspendierung für rechtmäßig. Im Falle Ägyptens läge ein Verstoß gegen die Interessen der Palästinenser vor. Die Lösung der Palästina-Frage gehört aber zu den Grundzielen der OIC.

Moinuddin erörtert jedoch nicht die Frage, ob die Charta, die keine Suspendierungsmöglichkeit enthält, dergestalt auszulegen ist, daß jeder islamische Staat das Recht auf Mitgliedschaft besitzt und eine Konfliktlösung innerhalb der Konferenz zu erfolgen hat. (Die Suspendierung Ägyptens wurde 1984 in Casablanca wieder aufgehoben.)

Die politische Ohnmacht der OIC in Krisensituationen wird durch den Iran-Irak-Krieg deutlich. Dies ist aber kein Phänomen der OIC, sondern ein allgemeines Problem, daß internationale Organisationen im Ernstfall nicht die Autorität haben, einen Konflikt zu verhindern bzw. zu lösen.

Im zweiten Teil seiner Arbeit befaßt sich Moinuddin mit den rechtlichen Rahmenbedingungen der islamischen Wirtschaftskooperation. Diese Zusammenarbeit verdient grundsätzlich Beachtung, da sie ein Beispiel für Kooperation unter Entwicklungsländern ist. Eine gewisse Einschränkung liegt darin, daß mit den Erdölstaaten als Entwicklungsländern eine atypische Situation vorliegt. Grundlage bildet das "General Agreement for Economic, Technical and Commercial Cooperation", dessen Ziel eine Einigkeit der OIC-Mitgliedstaaten über Ziele, Mittel und Bereiche der islamischen Wirtschaftskooperation ist. Das General Agreement ist somit eine Programm- und Willenserklärung zur Wirtschaftskoordination ohne unmittelbare Wirkung. Investitionen zwischen den islamischen Staaten sollen durch das "Agreement for Promotion, Protection and Guarantee of Investments" gefördert werden.

Dieses Abkommen entspricht im wesentlichen den üblichen Investitionsschutzverträgen, auch wenn die Formulierungen hinsichtlich der Enteignungsfrage den Standpunkten der Entwicklungsländer angenähert sind.

Es bleibt abzuwarten, ob eine verstärkte Zusammenarbeit der islamischen Staaten eintritt oder ob rein wirtschaftliche Erwägungen (z. B. bei Investitionen) am Ende doch schwerer als eine gemeinsame Religionszugehörigkeit wiegen. 